\title{
The properties of the Arithmetic Function as the Consecutive Sum of Digits in Natural Numbers
}

\author{
R. Maleki Chorei \\ Assistant Professor of Islamic Azad University of \\ Roudbar \\ Email : Dr.Maleki@iauroudbar.ac.ir
}

\begin{abstract}
In this paper defines the consecutive sum of the digits of a natural number, so far as it becomes less than ten, as an arithmetic function called $\psi$ and then introduces some important properties of this function by proving a few theorems in a way that they can be used as a powerful tool in many cases. As an instance, by introducing a test called $\psi$ test, it has been shown that we are able to examine many algebraic equalities in the form of $P=Q$ in which $P$ and $Q$ are arithmetic functions and to easily study many of the algebraic and diophantine equations in the domain of whole numbers. The importance of $\psi$ test for algebraic equalities can be considered equivalent to dimensional equation in physics relations and formulas. Additionally, this arithmetic function can also be useful in factorizing the composite odd numbers.
\end{abstract}

Keywords: consecutive sum of the digits, algebraic equations, diophantine equations, arithmetic functions

\section{Introduction}

By defining the consecutive sum of digits of a natural number as a $\psi$ function and by proving some theorems about their properties, we have been able to answer many questions about many types of algebraic and diophantine equations. In this paper, we have shown that we can prove some of the theorems of elementary number theory about diophantine equations by using $\psi$ as an arithmetic function. To do so, by introducing a test, called $\psi$ test, and notions as periodic matrix and periodic clock for any arithmetic function, we have studied the many types of algebraic equalities in the form of $P=Q$ in which $P$ and $Q$ can be any types of arithmetic functions. We have also indicated that we can use $\psi$ function to examine the factorability of numbers. This useful function can be utilized extensively in a way that we can use it in other systems.

\section{The $\psi$ Function}

\section{1- Definition of $\psi$ function and its properties}

If the sum of the digits constituting a natural number as $n$ in a decimal system with $\sum_{n}^{1}$, and the sum of digits constituting the number obtained from $\sum_{n}^{1}$ is represented 
by $\sum_{n}^{2}$ and also the sum of digits constituting the number obtained from $\sum_{n}^{2}$ is represented by $\sum_{n}^{3}$ and if we continue this process so far as we reach to $\sum_{n}^{n_{e}}$ which is a natural number less than 10 or a one-digit number, $\left(1 \leq \sum_{n}^{n e} \leq 9\right)$ then, in this case, we will define $\sum_{n}^{n_{e}}$ as an arithmetic function and it be represented by $\psi_{n}$.

$n \Rightarrow \sum_{n}^{1} \Rightarrow \sum_{n}^{2} \Rightarrow \ldots \Rightarrow \sum_{n}^{n e}=\psi_{n}$

The domain of definition of this function will be the natural numbers $(\mathbb{N})$ and its range in decimal system will be natural numbers less than 10.

Example 1.1- Calculate the value of $\psi$ function for the number $F=2,784,936$.

Answer: $\sum_{n}^{1}=2+7+8+4+9+3+6=39 \Rightarrow \sum_{n}^{2}=3+9=12 \Rightarrow$

$\sum_{n}^{3}=1+2=3 \Rightarrow \psi_{n}=3$

If some digits of a number are zero then we can conclude that it doesn't effect on the value of $\psi$.In the other words we always have $\psi_{F \times 10^{N}}=\psi_{F}$.

Theorem1.1- Whenever we assume $F=\sum_{i=1}^{n} F_{i}$, then we have $\psi_{F}=\psi_{\sum_{i=1}^{n} \psi_{F_{i}}}$.

Proof: First we propose $F=F_{1}+F_{2}, F_{1}=\overline{b_{1 N_{1}} \ldots b_{1 O}}$ and $F_{2}=\overline{c_{1 N_{1}} \ldots c_{1 O}}$ then we can write:

$F=F_{1}+F_{2} \Rightarrow \psi_{F}=\psi_{F_{1}+F_{2}}=\psi_{\left(b_{1 N_{1}} \times 10^{N_{1}}+\cdots+b_{10}\right)+\left(c_{1 N_{1}} \times 10^{N_{1}}+\cdots+c_{1 O}\right)}$

$=\psi_{\left(b_{1 N_{1}}+\cdots+b_{1 O}\right)+\cdots+\left(c_{1 N_{1}}+\cdots+c_{1 O}\right)}=\psi_{\sum_{F_{1}}^{1}+\sum_{F_{2}}^{1}}$

If we propose:

$\sum_{F_{1}}^{1}=b_{2 N_{2}} \times 10^{N_{2}}+\cdots+b_{2 O}$ and $\sum_{F_{2}}^{1}=c_{2 N_{2}} \times 10^{N_{2}}+\cdots+c_{2 O}$

Then we will have:

$\psi_{F}=\psi_{\sum_{F_{1}}^{1}+\sum_{F_{2}}^{1}}=\psi_{\left(b_{2 N_{2}} \times 10^{N_{2}}+\cdots+b_{2 O}\right)+\left(c_{2 N_{2}} \times 10^{N_{2}}+\cdots+c_{2 O}\right)}$

$=\psi_{\left(b_{2 N_{2}}+\cdots+b_{2 O}+\left(c_{2 N_{2}}+\cdots+c_{2 O}\right)\right.}=\psi_{\sum_{F_{1}}^{2}+\sum_{F_{2}}^{2}}$

Finally by continue the processes we reach to $\psi_{F}=\psi_{\sum_{F_{1}}^{n_{1} e}+\sum_{F_{2}}^{n_{2 e}}}=\psi_{\psi_{F_{1}}+\psi_{F_{2}}}$.

By generalized above result we can conclude if $F=\sum_{i=1}^{n} F_{i}$ then we have

$\psi_{F}=\psi_{\sum_{i=1}^{n} \psi_{F_{i}}}$

Example1.2- If $F_{1}=821, F_{2}=2381$, then calculate the $\psi$ value for the $F_{1}+F_{2}$. 
Answer: $\psi_{821+2381}=\psi_{\psi_{821}+\psi_{2381}}=\psi_{2+5}=\psi_{7}=7$

Theorem1.2- For every arithmetic polynomial function as $f(n)=a_{m} n^{m}+$ $a_{m-1} n^{m-1}+\cdots++a_{0}$, the values of $f(n)$ and $f(n+9 k)$ are congruent modulo $9(k$ is a whole number).

Proof: Based on the general form of an arithmetic polynomial function, we will have:

$f(n+9 k)-f(n)=$

$a_{m}\left[(n+9 k)^{m}-n^{m}\right]+a_{m-1}\left[(n+9 k)^{m-1}-n^{m-1}\right]+\cdots+a_{1}[(n+9 k)-n]$

Based on the algebraic identity of $x^{k}-y^{k}=(x-y)\left(x^{k-1}+x^{k-2} y+\cdots+y^{k-1}\right)$, we will have $f(n+9 k)-f(n)=a_{m}\left[(9 k) k_{m}\right]+a_{m-1}\left[(9 k) k_{m-1}\right]+\cdots+a_{1} \times 9 k=$ $9 k\left(a_{m} k_{m}+a_{m-1} k_{m-1}+\cdots+a_{1}\right) \Rightarrow f(n+9 k) \equiv f(n) \bmod 9$.

Theorem 1.3- For every natural number as a multiple of 9, the value of $\psi$ equals to 9.

Proof: Suppose $F=\overline{a_{N_{1}} a_{N_{1}-1} \ldots a_{1} a_{0}}$, since $F$ is assumed as a multiple of 9, then we get:

$\sum_{F}^{1}=\sum_{i=0}^{N_{1}} a_{i}=9 k_{1}=\overline{b_{N_{2}} b_{N_{2}-1} \ldots b_{1} b_{0}} \Rightarrow \sum_{F}^{2}=\sum_{i=0}^{N_{2}} b_{i}=9 k_{2} \Rightarrow \cdots$

Since we have:

$$
k_{1}>k_{2}>\cdots
$$

Therefore at the end, we will reach to a case in which $\sum_{F}^{n e}=9$ that is the $\psi$ value for number $F$.

Theorem 1.4- In any arithmetic polynomial function as $f(n)$, the values of $\psi_{f(n+9 k)}$ and $\psi_{f(n)}$ are equal.

Proof: According to theorem (1.2), we have:

$f(n+9 k)=f(n) \bmod 9 \Rightarrow f(n+9 k)=f(n)+9 k$

From theorem (1.1), we get $\psi_{f(n+9 k)}=\psi_{\psi_{f(n)}+\psi_{9 k}}$.

Also from theorem(1.3), we get $\psi_{9 k}=9$, therefore we will have:

$$
\psi_{f(n+9 k)}=\psi_{f(n)+9}
$$

Since $\psi_{f(n)}$ is less than ten, we will have:

$\psi_{f(n)}=1 \Rightarrow \psi_{f(n)}+9=10 \Rightarrow \psi_{f(n)+9}=1=\psi_{f(n)}$

$\psi_{f(n)}=2 \Rightarrow \psi_{f(n)}+9=11 \Rightarrow \psi_{\psi f(n)+9}=2=\psi_{f(n)}$ 
$\psi_{f(n)}=9 \Rightarrow \psi_{f(n)}+9=18 \Rightarrow \psi_{f(n)+9}=9=\psi_{f(n)}$

Therefore, in the general case, we get $\psi_{f(n)+9}=\psi_{f(n)}$, then according to (1), we can conclude that $\psi_{f(n+9 k)}=\psi_{f(n)}$

Result 1.1- Whenever the two natural numbers as $F_{1}$ and $F_{2}$ are congruent modulo 9, then the $\psi$ values will be equal and vice versa. In other words:

$F_{2} \equiv F_{1} \bmod 9 \Leftrightarrow \psi_{F_{1}}=\psi_{F_{2}}$

Result 1.2 -The difference of every number from the sum of its digits or from the value of its $\psi$ is divisible by 9. This is because, according to the definition of $\psi$ function, we have $\psi_{F}=\psi_{\sum_{F}^{1}}$ thus according to the result (1.1) of the theorem(1.4), we can write:

$$
F-\sum_{F}^{1}=9 k \text { or } F \equiv \sum_{F}^{1} \bmod 9
$$

We also have :

$\psi_{F}=\psi_{\psi_{F}} \Rightarrow F \equiv \psi_{F} \bmod 9$

According to theorem (1.4) in any arithmetic polynomial function as $f(n)$, the values of $\psi$ will be repeated for any 9 consecutive values of $n$. In other words, the values of $\psi$ for 9 consecutive values of $n$, will have a period for that arithmetic function in question. The $\psi$ values of any period of an arithmetic polynomial function is represented in the form of a $1 \times 9$ matrix and is called a periodic matrix of $f(n)$ as a arithmetic polynomial function and is denoted by $T_{f(n)}$. In other hand we have:

$T_{f(n)}=\left[\psi_{f(1)}, \psi_{f(2)}, \ldots, \psi_{f(9)}\right]$

Therefore we can show the periodic matrix of any arithmetic function in the form of a clockwise periodic clock as follows which is called the periodic clock of the arithmetic function in question. Contractually it is represented by $\vec{\odot}_{f(n)}$.

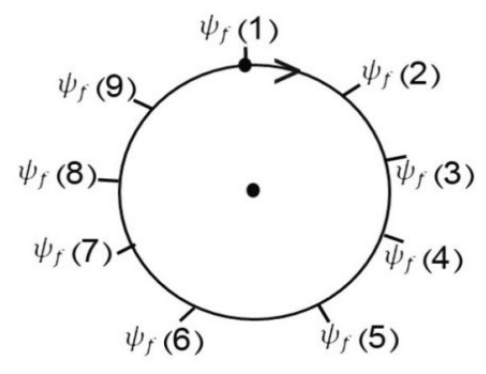

The set which includes different values of $\psi$ related to a periodic matrix is denoted by $H_{f_{(n)}}$. Therefore, the maximum number of members of $H_{f_{(n)}}$ is equal to 9.

As an instance, for the arithmetic function $f(n)=n^{2}$ we have: 
$T_{f(n)}=\left[\psi_{f(1)}, \psi_{f(2)}, \psi_{f(3)}, \psi_{f(4)}, \psi_{f(5)}, \psi_{f(6)}, \psi_{f(7)}, \psi_{f(8)}, \psi_{f(9)}\right]$

$=[1,4,9,7,7,9,4,1,9]$

Therefore, the periodic matrix of the arithmetic function of $f(n)$ will be as:

$T_{f(n)}=[1,4,9,7,7,9,4,1,9] \Rightarrow H_{f(n)}=\{1,4,7,9\}$

As a result, the periodic clock of the arithmetic function will be as follows:

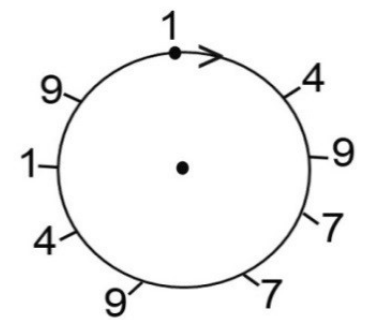

Therefore, all the natural numbers can be divided in 9 arithmetic series in a way that the numbers of each arithmetic series contains the same periodic matrix and clock. In other words, the natural numbers can be partitioned into 9 sets, as shown below, in a way that the $\psi$ value of the members of each set will be the same.

$S_{i}=\{i+9 k \mid k \in W\} \quad, \quad i \in N, \quad 1 \leq i \leq 9$

(W and $N$ represents the whole numbers and natural number respectively)

In a special case, if we assume $f(n)=k n$ and $k \neq 3 m$, we will always get:

$\vec{\odot}_{f(n)}=\vec{\bigodot}_{f(n)+C \quad \text { (c is a whole number) }}$

As an example, we have:

$\left\{\begin{array}{l}T_{7 n}=[7,5,3,1,8,6,4,2,9] \\ T_{7 n+2}=[9,7,5,3,1,8,6,4,2]\end{array} \quad \Rightarrow \quad \vec{\odot}_{7 n}=\vec{\odot}_{7 n+2}\right.$

Note 1.1-For two arithmetic functions in which $(n)=g(n)+9 k$, we will always have:

$T_{f(n)}=T_{g(n)} \quad, \quad H_{f(n)}=H_{g(n)} \quad, \quad \vec{\odot}_{f(n)}=\vec{\odot}_{g(n)}$

Note 1.2- Whenever $f(n)=a n+c, g(n)=b n+d$ and $\psi_{\psi_{a}+\psi_{b}}=9$ then the arithmetic functions of $f(n)$ and $g(n)$ will have periodic clocks with the same clockwise and counterclockwise sequences. This means that they are inverses of each other. In other words, in this case we will have:

$H_{f(n)}=H_{g(n)}, \vec{\odot}_{f(n)}=\overleftarrow{\odot}_{g(n)}, T_{f(n)+g(n)}=[\overline{9}]_{9}$

As an instance, for two arithmetic functions as $f(n)=2 n+3$ and $g(n)=7 n+5$, we have:

$a=2, b=7 \Rightarrow \psi_{\psi_{a}+\psi_{b}}=9 \Rightarrow \vec{\odot}_{f(n)}=\overleftarrow{\odot}_{g(n)}$ 
The above point can be observed in the list of periodic matrixes as below:

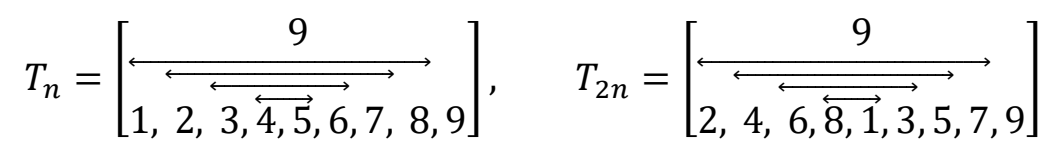

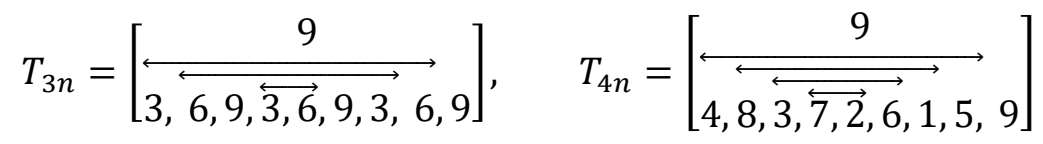

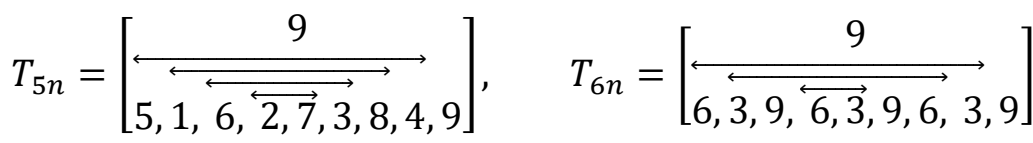

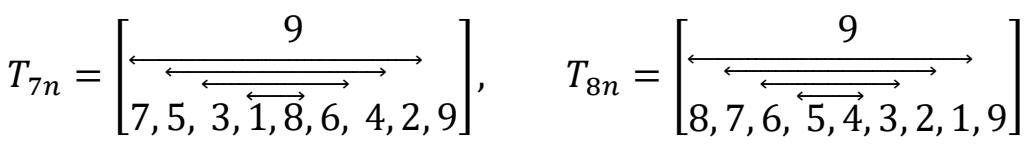

Theorem 1.5- If we assume $F=\Pi_{i=1}^{n} F_{i}$, then we have $\psi_{F}=\psi_{\Pi_{i=1}^{n} \psi_{F(i)}}$.

Proof: First for $n=2$ and from theorem(1.1) we have

$\psi_{F}=\psi_{F_{1} \times F_{2}}=\psi_{F_{1} \underbrace{(1+1+000+1)}_{F_{2} \text { times }}}=\psi_{\underbrace{\left(F_{1}+F_{1}+000+F_{1}\right)}_{F_{2} \text { times }}}=\psi_{F_{F_{1} \text { times }}^{\psi_{\left.F_{1}+\psi_{F_{1}}+000+\psi_{F_{1}}\right)}}}^{\underbrace{}_{F_{2}} \psi_{F_{1}}}$

Whereas can be written:

$F_{2} \psi_{F_{1}}-\psi_{F_{2}} \psi_{F_{1}}=\psi_{F_{1}}\left(F_{2}-\psi_{F_{2}}\right)$

But according to the result( 1) of the theorem (4), we have:

$F_{2} \equiv \psi_{F_{2}} \bmod 9 \Rightarrow F_{2}-\psi_{F_{2}}=9 k$

Thus, from (2) we will get $F_{2} \psi_{F_{1}}-\psi_{F_{1}} \psi_{F_{2}}=9 k \psi_{F_{1}}$.

Therefore, according to (1) and the result of the theorem (1.4) we have:

$F_{2} \psi_{F_{1}}=\psi_{\psi_{F_{1}} \times \psi_{F_{2}}} \Rightarrow \psi_{F_{1} \times F_{2}}=\psi_{\psi_{F_{1}} \times \psi_{F_{2}}}$

As a result, by generalizing the result of the above proof we will get:

$\psi_{\Pi_{i=1}^{n} F_{i}}=\psi_{\Pi_{i=1}^{n} \psi_{F(i)}}$

The important results that we can conclude from theorems (1.1) and(1. 5) are as follows:

1. $F=F_{1}+F_{2}+\cdots+F_{n} \Rightarrow \psi_{F}=\psi_{\sum_{i=1}^{n} \psi_{F_{i}}} \Rightarrow F \equiv \sum_{i=1}^{n} \psi_{F_{i}} \bmod 9$

2. $F=F_{1} \times F_{2} \times \ldots \times F_{n} \Rightarrow \psi_{F}=\psi_{\Pi_{i=1}^{n} \psi_{F_{i}}} \Rightarrow F \equiv \Pi_{i=1}^{n} \psi_{F_{i}} \bmod 9$

3. In order to calculate the $\psi$ value for expressions in the form of $\left(\sum_{i=1}^{m} F_{i}\right)^{n}$ we will do as below: 
$\psi_{\left(\sum_{i=1}^{m} F_{i}\right)^{n}}=\psi_{\left(\psi_{\sum_{i=1}^{m} \psi_{F_{i}}}\right)^{n}}$

4. In order to calculate the $\psi$ value for expressions in the form of $\left.\Pi_{i=1}^{m} F_{i}\right)^{n}$ we will do as below:

$\left.\psi_{\left(\Pi_{i=1}^{m} F_{i}\right)^{n}}=\psi_{\left(\psi_{\Pi_{i=1}}^{m} \psi_{F_{i}}\right.}\right)^{n}$

5. In order to calculate the $\psi$ value of the arithmetic function as $f(n)=A^{n}$ we will do as below:

$\psi_{f(n)}=\psi_{A^{n}}=\psi_{\underbrace{}_{\text {times }} \times{ }_{\text {time }} \times{ }^{\prime}}=\psi_{\underbrace{\psi_{A} \times \psi_{A} \times \ldots \times \psi_{A}}_{n \text { times }}}=\psi_{\left(\psi_{A}\right)^{n}}$

6. In order to calculate the $\psi$ value for expressions in the form of $\Pi_{i=1}^{n} F_{i}{ }^{m_{i}}$ we will do as below:

$\psi_{\Pi_{i=1}^{n} F_{i}}^{m_{i}}=\psi_{\Pi_{i=1}^{n}\left(\psi_{F_{i}}\right)^{m_{i}}}$

7. Whenever $A_{1} \equiv B_{1} \bmod 9, A_{2} \equiv B_{2} \bmod 9, \ldots, A_{n} \equiv B_{n} \bmod 9$ then according to the properties of the congruences and the properties of the $\psi$ function, we will easily get:

$\sum_{i=1}^{n} \psi_{A_{i}} \equiv \sum_{i=1}^{n} \psi_{B_{i}} \bmod 9$ and $\prod_{i=1}^{n} \psi_{A_{i}} \equiv \prod_{i=1}^{n} \psi_{B_{i}} \bmod 9$

8. For every natural number constituted by linear combination of n natural numbers as $F_{1}^{m_{1}}$ to $F_{n}^{m_{n}}$, on the condition that $k_{i}$ and $m_{i}$ are non-negative integer numbers, we will do as follows:

$\psi_{F_{i}} m_{i}=\psi_{F_{i} m_{i}}=\psi_{\left(\psi_{F_{i}}\right)^{m_{i}}} \Rightarrow \psi_{k_{i} F_{i}} m_{i}=\psi_{k_{i}\left(\psi_{F_{i}}\right)^{m_{i}}} \Rightarrow \psi_{\sum_{i=1}^{n} k_{i} F_{i}} m_{i}=\psi_{\sum_{i=1}^{n} k_{i}\left(\psi_{F_{i}}\right)^{m_{i}}}$

Theorem 1.6- In the arithmetic exponential function as $f(n)=A^{6 n}$, the value of $\psi_{f(n)}$ will always be equal to 1 if $A$ is not a multiple of 3.

Proof: Since it is assumed that A is not a multiple of 3 , then for one-digit natural values of $A$ we have:

$$
\begin{aligned}
& f(n)=1^{6 n} \Rightarrow \psi_{f(n)}=\psi_{1^{6 n}}=\psi_{1}=1 \\
& f(n)=2^{6 n} \Rightarrow \psi_{f(n)}=\psi_{2^{6 n}}=\psi_{\left(2^{6}\right)^{n}}=\psi_{\left(\psi_{64}\right)^{n}}=\psi_{\left(\psi_{10}\right)^{n}}=\psi_{1^{n}}=1 \\
& f(n)=4^{6 n} \Rightarrow \psi_{f(n)}=\psi_{4^{6 n}}=\psi_{\left(4^{6}\right)^{n}}=\psi_{\left(\psi_{4}\right)^{n}}=\psi_{\left(\psi_{4096}\right)^{n}}=\psi_{\left(\psi_{19}\right)^{n}}=\psi_{1^{n}}=1 \\
& f(n)=5^{6 n} \Rightarrow \psi_{f(n)}=\psi_{5^{6 n}}=\psi_{\left(5^{6}\right)^{n}}=\psi_{\left(\psi_{5^{6}}\right)^{n}}=\psi_{\left(\psi_{15625}\right)^{n}}=\psi_{\left(\psi_{19}\right)^{n}}=\psi_{1^{n}}=1 \\
& f(n)=7^{6 n} \Rightarrow \psi_{f(n)}=\psi_{7^{6 n}}=\psi_{\left(7^{6}\right)^{n}}=\psi_{\left(\psi_{\left.7^{6}\right)^{n}}\right.}=\psi_{\left(\psi_{117649}\right)^{n}}=\psi_{\left(\psi_{28}\right)^{n}}=\psi_{1^{n}}=1 \\
& f(n)=8^{6 n} \Rightarrow \psi_{f(n)}=\psi_{8^{6 n}}=\psi_{\left(8^{6}\right)^{n}}=\psi_{\left(\psi_{8^{6}}\right)^{n}}=\psi_{\left(\psi_{262144}\right)^{n}}=\psi_{\left(\psi_{19}\right)^{n}}=\psi_{1^{n}}=1
\end{aligned}
$$


Therefore, in the general case, we have:

$A \in\{1,2,4,5,7,8\} \Rightarrow \psi_{f(n)}=\psi_{(A)^{6 n}}=1$.

Theorem 1.7- In an arithmetic exponential function as $f(n)=A^{n}$ in which $A$ is a constant natural number, the periodic matrix of the function, contain 6 members. In other words, $T_{f(n)}$ contains 6 values of the consecutive values of $\psi_{f(n)}$.

Proof: First we assume $n=6 k+B$ in which $B$ is a non-negative integer number and $0 \leq B \leq 9$.

In this case we will have:

$f(n)=A^{n}=A^{6 k+B}=A^{6 k} \times A^{B} \Rightarrow \psi_{f(n)}=\psi_{A^{n}}=\psi_{A^{6 k} \times A^{B}}=\psi_{\psi_{A^{6 K}} \times \psi_{A^{B}}}$

Therefore, according to theorem (6) we get:

$\psi_{A^{6 K}}=1 \Rightarrow \psi_{f(n)}=\psi_{1 \times \psi_{A^{B}}}=\psi_{\psi_{A^{B}}}=\psi_{A^{B}}$

Thus, $T_{f(n)}$ of an arithmetic exponential function, as $f(n)=A^{6 n}$, contains 6 consecutive values of $\psi_{f(n)}$ values. In other words, for any exponential function in the form of $f(n)=A^{6 n}$ we have $\psi_{f(n+6 k)}=\psi_{f(n)}$.

Theorem 1.8-For both of the non-negative integer numbers as $A$ and $B$, the values of $\psi_{A^{B}}$ and $\psi_{\left(A+9 K_{1}\right)^{\left(B+6 K_{2}\right)}}$ are equal $\left(K_{1}, K_{2}\right.$ are non-negative integer numbers).

Proof: If we assume $\left(A+9 K_{1}\right)^{\left(B+6 K_{2}\right)}=M^{N}$, from theorem (1.4 $)$ we have:

$M^{N}=\left(A+9 K_{1}\right)^{N} \Rightarrow \psi_{\left(A+9 K_{1}\right)^{N}}=\psi_{A^{N}}$

Also from theorem( 1.6) we have:

$M^{N}=M^{B+6 K_{2}} \Rightarrow \psi_{M^{B+6 K_{2}}}=\psi_{M^{B}}$

Therefore, from (1) and (2) we can conclude:

$\left(A+9 K_{1}\right)^{\left(B+6 K_{2}\right)}=\psi_{A^{B}}$.

Note that in the proof above $A$ and $B$ do not have to be less than ten and that to calculate the $\psi$ value for the numbers in the form of $M^{N}$, for easier calculation, $A$ and $B$ are better to be as less than ten.

Example1.3- Calculate the value of $\psi$ for the number $F=2387^{473}$.

Answer: $F=2387^{473}=M^{N} \Rightarrow M=2387 \Rightarrow \sum_{M}^{1}=20=2 \times 9+2 \Rightarrow A=2$

$N=473=$ odd number $\Rightarrow \sum_{N}^{1}=14=2 \times 6+2 \Rightarrow B=2 \Rightarrow \psi_{F}=\psi_{2^{2}}=\psi_{4}=4$ 
Example 1.4- Show that if $p$ is a prime number in the form of $6 n+1$, then we have $a^{p-1} \equiv 1 \bmod 9 p$.

Answer: From Fermat's little theorem we have:

$a^{p-1} \equiv 1 \bmod p \Rightarrow a^{p-1}=1+k p$

$p=6 n+1 \Rightarrow a^{6 n}=1+k p \Rightarrow \psi_{a^{6 n}}=\psi_{\psi_{1}+\psi_{k p}} \Rightarrow 1=\psi_{1+\psi_{k p}}$

Therefore, according to theorem (1.4) we get:

$\psi_{k p}=9 \Rightarrow k=9 m \Rightarrow a^{p-1} \equiv 1 \bmod 9 p$

\section{2-Some notes on the calculation the $\psi$ value of the exponential numbers}

1. To calculate the $\psi$ value of natural numbers in the form of $F=A^{\prod_{i=1}^{n_{1}} B_{i}}$ we will do as below: $\left(A \neq 3 \mathrm{~m}\right.$ and the values of $a, b_{(i)}$, and $b$ are less than 10)

$F=(a+9 k)^{\prod_{i=1}^{n}\left(b_{(i)}+6 k_{b_{(i)}}\right)} \Rightarrow \psi_{F}=\psi_{a^{\Pi_{i=1}^{n} b_{(i)}}}=\psi_{a^{6 k+b)}}=\psi_{a^{b}}$

As an example, to calculate $\psi$ of the number $F=34832^{13 \times 23 \times 49 \times 34}$ we will do as below:

$3+4+8+3+2=20=2 \times 9+2 \Rightarrow F=(2+2 \times 9)^{(2 \times 6+1)(3 \times 6+5)(6 \times 8+1)(5 \times 6+5)}$

$\Rightarrow \psi_{F}=\psi_{2^{1 \times 5 \times 1 \times 5}}=\psi_{2^{25}}=\psi_{2^{4 \times 6+1}}=\psi_{2^{1}}=2$

2. To calculate $\psi$ of the numbers in the form of $F=\prod_{i=1}^{n} A_{i}^{B_{i}}$ in which none of the values of $A_{i}$ are not multiple of 3 , we will do as below:

$F=\prod_{i=1}^{n}\left(a_{i}+9 k_{i}\right)^{b_{i}+6 k_{i}^{\prime}} \Rightarrow \psi_{F}=\psi_{\prod_{i=1}^{n} a_{i}^{b_{i}}}=\psi_{\prod_{i=1}^{n} \psi_{a_{i}}^{b_{i}}}$

As an example, for calculate $\psi$ of the number as $F=334^{17} \times 42743^{94}$ we will do as below:

$F=\left(9 K_{1}+1\right)^{6 K_{1}^{\prime}+5} \times\left(9 K_{2}+2\right)^{6 K_{2}^{\prime}+4} \Rightarrow \psi_{F}=\psi_{1^{5} \times 2^{4}}=\psi_{16}=7$

3. If in $F=\prod_{i=1}^{n} A_{i}^{B_{i}}$ at least one of the values of $A_{i}$ is a multiple of 3 , we get:

$A_{i}=3 m \quad \Rightarrow \quad \psi_{F} \in\{3,6,9\}$

4. To calculate $\psi$ of the numbers in the form of $F=A^{B^{m}}$ in which $A$ is not a multiple of 3, we will do as below:

$$
F=A^{B^{m}}=\left(9 k_{1}+a\right)^{\left(6 k_{1}+b\right)^{m}}=a^{6 k_{2}+b^{m}} \Rightarrow \psi_{F}=\psi_{a^{b^{m}}}=\psi_{a^{6 n+c}}=\psi_{a^{c}}
$$

As an example, for calculate $\psi$ of the number $F=587^{315^{3}}$ we will do as below: 
$F=(9 k+2)^{\left(6 k^{\prime}+3\right)^{3}}=(9 k+2)^{6 k^{\prime \prime}+3^{3}} \Rightarrow \psi_{F}=\psi_{2^{3^{3}}}=\psi_{2^{4 \times 6+3}}=\psi_{2^{3}}=8$

5. To calculate $\psi$ of the exponential function $f(n)=A^{B n}$ we will do as below:

$f(n)=A^{B n}=\left(9 k_{1}+a\right)^{\left(6 k_{2}+b\right) n} \cdot(1 \leq b \leq 6,1 \leq a \leq 9)$

$\Rightarrow \psi_{f(n)}=\psi_{a^{b n}}=\psi_{\left(\psi_{a}{ }^{b}\right)^{n}}$

As an example, for the arithmetic function $f(n)=17^{11 n}$ we get:

$f(n)=(1 \times 9+8)^{(1 \times 6+5) n} \Rightarrow \psi_{f(n)}=\psi_{8^{5 n}}=\psi_{\left(8^{5}\right)^{n}}=\psi_{\left(8^{2} \times 8^{2} \times 8\right)^{n}}$

$=\psi_{\left(\psi_{8^{2}} \times \psi_{8^{2}} \times \psi_{8}\right)^{n}}=\psi_{(1 \times 1 \times 8)^{n}}=\psi_{8^{n}} \Rightarrow T_{8^{n}}=[\overline{1,8}]_{3}$

6. To calculate the arithmetic functions in the form of $f(n)=a^{b^{n}}$, it is better to do as

$\psi_{a^{b^{n}}}=\psi_{a^{\left(6 k_{1}+c\right)^{n}}}=\psi_{a^{6 k_{2}+c^{n}}}=\psi_{a^{c^{n}}}=\psi_{a^{6 k_{3}+d}}=\psi_{a^{d}}$.

As an example, to calculate the value of $\psi$ of the number $5^{8^{4}}$ we will do as below:

$\psi_{5^{8^{4}}}=\psi_{5^{(6+2)^{4}}}=\psi_{5^{6 k+2^{4}}}=\psi_{5^{2^{4}}}=\psi_{5^{16}}=\psi_{5^{2 \times 6+4}}=\psi_{5^{4}}=\psi_{625}=\psi_{4}=4$

Note1.3-The periodic clocks of arithmetic functions of $A^{n}, A^{n+B}, k \times A^{n}$, and, $k \times$ $A^{n+B}$ are always similar. In other words, we have: (when $k \neq 3 m$ )

$\vec{\bigodot}_{A^{n}}=\vec{\bigodot}_{A^{n+B}}=\vec{\bigodot}_{k \times A^{n}}=\vec{\bigodot}_{k \times A^{n+B}}$

As an example, we have:

$T_{2^{n}}=[2,4,8,7,5,1] \Rightarrow T_{2^{n+3}}=[7,5,1,2,4,8] \Rightarrow T_{5 \times 2^{n}}=[1,2,4,8,7,5]$

$\Rightarrow T_{5 \times 2^{n+3}}=[8,7,5,1,2,4] \Rightarrow \vec{\odot}_{2^{n}}=\vec{\odot}_{2^{n+3}}=\vec{\bigodot}_{5 \times 2^{n}}=\vec{\bigodot}_{5 \times 2^{n+3}}$

Theorem 1. 9- Whenever $A$ and $B$ are two natural numbers and $A>B$, then we always get $\psi_{A-B}=\psi_{\left(\psi_{A}-\psi_{B}\right)+9}$.

Proof: According to theorems (1.1) and (1.4) and the definition of $\psi$, we have:

State 1: If $\psi_{A}>\psi_{B}$

$A>B \Rightarrow A-B=C>0 \Rightarrow A=B+C \Rightarrow \psi_{A}=\psi_{B+C} \Rightarrow \psi_{\psi_{A}}=\psi_{\psi_{B+} \psi_{C}}$

$=\psi_{\psi_{B}}+\psi_{\psi_{C}}$

$\psi_{A}>\psi_{B} \Rightarrow \psi_{\psi_{C}}=\psi_{\psi_{A}}-\psi_{\psi_{B}}=\psi_{A}-\psi_{B}=\psi_{\psi_{A-} \psi_{B}}$

$\Rightarrow \psi_{C}=\psi_{\psi_{A-} \psi_{B}} \Rightarrow \psi_{A-B}=\psi_{\psi_{A-} \psi_{B}}=\psi_{\left(\psi_{A}-\psi_{B}\right)+9}$

State 2: If $\psi_{A}<\psi_{B}$, then we have $\psi_{A}+9>\psi_{B}$ therefore according to state (1), we can conclude: 
$\psi_{A-B}=\psi_{(A-B)+9}=\psi_{(A+9)-B}=\psi_{\psi_{A+9}-\psi_{B}}=\psi_{\left(\psi_{A}+\psi_{9}\right)-\psi_{B}}=\psi_{\left(\psi_{A}-\psi_{B}\right)+9}$.

Example 1.5-Calculate the value of $\psi$ of the number $F=344^{235}-563^{1796}$.

$F=\left(9 k_{1}+4\right)^{6 k_{2}+1}-\left(9 k_{3}+5\right)^{6 k_{4}+3}$

$\Rightarrow \psi_{F}=\psi_{\left(\psi_{\left.4^{1}-5^{3}\right)+9}\right.}=\psi_{\left(\psi_{4^{1}}-\psi_{5^{3}}\right)+9}=\psi_{5}=5$

Note 1.4- Whenever $A=0$, then we will define $\psi_{A}$ equal to 9. In other words, we will have:

$A=0 \Rightarrow \psi_{A}=9$

Therefore, when we have $\psi_{M}=\psi_{N}$ it means that $\psi_{M-N}=9$.

One of the important conclusions that we can reach from theorem(1.9) is as follows:

$\psi_{(a-b)^{2}}=\psi_{\left(\psi_{a}-\psi_{b}+9\right)^{2}}$ or $\psi_{(a-b)^{n}}=\psi_{\left(\psi_{a}-\psi_{b}\right)^{n}+9}$

We should note that, according to the identity $(x+9)^{n}$ and theorem (1.4), we always have:

$\psi_{(x+9)^{n}}=\psi_{x^{n}+9}=\psi_{x^{n}}$

Then in the general case we get:

$\psi_{(a-b)^{n}}=\psi_{\left(\psi_{a}-\psi_{b}\right)^{n}+9}$ or $\quad \psi_{(a-b)^{n}}=\psi_{\left(\psi_{a}-\psi_{b}+9\right)^{n}}$

Table1.1- The list of the $\psi$ values for the arithmetic polynomial base functions and arithmetic exponential base functions .

\begin{tabular}{|l|l|}
\hline Arithmetic function & $\begin{array}{l}\text { Periodic matrix of the arithmetic function } \\
\text { of } f(n)\end{array}$ \\
\hline$f(n)=(1)^{n}$ & $T=[\overline{1}]_{9}$ \\
\hline$f(n)=(2)^{n}$ & $T=[2,4,8,7,5,1]$ \\
\hline$f(n)=(4)^{n}$ & $\begin{array}{l}T=[\overline{4,7,1}]_{2} \\
f(n)=4^{3 n+1} \rightarrow \psi=4 \\
f(n)=4^{3 n-1} \rightarrow \psi=7 \\
f(n)=4^{3 n} \rightarrow \psi=1\end{array}$ \\
\hline$f(n)=(5)^{n}$ & $T=[5,7,8,4,2,1]$ \\
\hline$f(n)=(7)^{n}$ & $\begin{array}{l}T=[\overline{7,4,1}]_{2} \\
f(n)=7^{3 n+1} \rightarrow \psi=7 \\
f(n)=7^{3 n-1} \rightarrow \psi=4\end{array}$ \\
\hline
\end{tabular}




\begin{tabular}{|c|c|}
\hline & $f(n)=7^{3 n} \rightarrow \psi=1$ \\
\hline$f(n)=(8)^{n}$ & $\begin{array}{l}T=[\overline{8,1,8}]_{2} \\
f(n)=8^{2 k-1} \rightarrow \psi=8 \\
f(n)=8^{2 k} \rightarrow \psi=1\end{array}$ \\
\hline $\begin{array}{l}f(n)=(3)^{n} \\
f(n)=(6)^{n} \\
f(n)=(9)^{n}\end{array}$ & $T=[\overline{9}]_{9}, n \geq 2$ \\
\hline$f(n)=(n)^{1}$ & $T=[1,2,3,4,5,6,7,8,9]$ \\
\hline$f(n)=(n)^{2}$ & $T=[1,4,9,7,7,9,4,1,9]$ \\
\hline$f(n)=(n)^{3}$ & $\begin{array}{l}T=[\overline{1,8,9}]_{3} \\
f(n)=(3 n+1)^{3} \rightarrow \psi=1 \\
f(n)=(3 n-1)^{3} \rightarrow \psi=8 \\
f(n)=(3 n)^{3} \rightarrow \psi=9\end{array}$ \\
\hline$f(n)=(n)^{4}$ & $T=[1,7,9,4,4,9,7,1,9]$ \\
\hline$f(n)=(n)^{5}$ & $T=[1,5,9,7,1,9,4,8,9]$ \\
\hline$f(n)=(n)^{6}$ & $\begin{array}{l}T=[\overline{1,1,9}]_{3} \\
f(n)=(3 n \pm 1)^{6 k} \rightarrow \psi=1 \\
f(n)=(6 n)^{6 k} \rightarrow \psi=9\end{array}$ \\
\hline
\end{tabular}

Table1.2- The list of periodic matrixes of the arithmetic functions as $f(n)=$ $(3 k)^{n}$ and, $f(n)=(3 k \pm 1)^{n}$

$$
\begin{aligned}
& f(k)=3 k-1 \rightarrow T=[\overline{2,5,8}]_{3} \\
& f(k)=3 k+1 \rightarrow T=[\overline{4,7,1}]_{3} \\
& f(k)=3 k \rightarrow T=[\overline{3,6,9}]_{3} \\
& f(k)=(3 k-1)^{2} \rightarrow T=[\overline{4,7,1}]_{3} \\
& f(k)=(3 k+1)^{2} \rightarrow T=[\overline{4,7,1}]_{3} \\
& f(k)=(3 k)^{2} \rightarrow T=[\overline{9}]_{9} \\
& f(k)=(3 k-1)^{3} \rightarrow T=[\overline{8}]_{9} \\
& f(k)=(3 k+1)^{3} \rightarrow T=[\overline{1}]_{9} \\
& f(k)=(3 k)^{2} \rightarrow T=[\overline{9}]_{9} \\
& f(k)=(3 k-1)^{4} \rightarrow T=[\overline{4,7,1}]_{3}
\end{aligned}
$$




\begin{tabular}{|l|}
\hline$f(k)=(3 k+1)^{4} \rightarrow T=[\overline{4,7,1}]_{3}$ \\
$f(k)=(3 k)^{4} \rightarrow T=[\overline{9}]_{9}$ \\
\hline$f(k)=(3 k-1)^{5} \rightarrow T=[\overline{2,5,8}]_{3}$ \\
$f(k)=(3 k+1)^{5} \rightarrow T=[\overline{4,7,1}]_{3}$ \\
$f(k)=(3 k)^{5} \rightarrow T=[\overline{9}]_{9}$ \\
\hline$f(k)=(3 k-1)^{6} \rightarrow T=[\overline{1}]_{9}$ \\
$f(k)=(3 k+1)^{6} \rightarrow T=[\overline{1}]_{9}$ \\
$f(k)=(3 k)^{6} \rightarrow T=[\overline{9}]_{9}$ \\
\hline$f(k)=(3 k-1)^{7} \rightarrow T=[\overline{2,5,8}]_{3}$ \\
$f(k)=(3 k+1)^{7} \rightarrow T=[\overline{4,7,1}]_{3}$ \\
$f(k)=(3 k)^{7} \rightarrow T=[\overline{9}]_{9}$
\end{tabular}

Table1.3- The list of periodic matrixes of $T_{a^{n}+b^{n}}$

$$
\begin{aligned}
& T_{1^{n}+2^{n}}=[3,5,9,8,6,2], T_{1^{n}+4^{n}}=[\overline{5,8,2}]_{6^{\prime}}, T_{1^{n}+5^{n}}=[6,8,95,3,2], T_{1^{n}+7^{n}}=[\overline{8,5}, 2] \\
& T_{1^{n}+8^{n}}=[\overline{9,2}]_{6}, T_{2^{n}+2^{n}}=[4,8,7,5,1,2], T_{2^{n}+4^{n}}=[6,2,9,2,3,2], T_{2^{n}+5^{n}}=[\overline{7.2}] \\
& T_{2^{n}+7^{n}}=[9,8,9,5,9,2], T_{2^{n}+8^{n}}=[1,5,7,8,4,2], T_{4^{n}+4^{n}}=[\overline{8,5}, 2]_{6} \\
& T_{4^{n}+5^{n}}=[9,5,9,8,9,2], T_{4^{n}+7^{n}}=[\overline{2}]_{6}, T_{4^{n}+8^{n}}=[3,8,9,5,6,2], \\
& T_{5^{n}+7^{n}}=[3,2,9,2,6,2], T_{5^{n}+8^{n}}=[4,8.7,5,1,2], T_{5^{n}+5^{n}}=[1,5,7,8,4,2] \\
& T_{5^{n}+7^{n}}=[3,2,9,2,6,2], T_{5^{n}+8^{n}}=[4,8.7,5,1,2], T_{7^{n}+7^{n}}=[8,5,2] \\
& T_{7^{n}+8^{n}}=[6,5,9,8,3,2], T_{8^{n}+8^{n}}=[\overline{7,2}]_{6}
\end{aligned}
$$

Table1.4- The list of periodic matrixes of $T_{a^{n}-b^{n}}$

$$
\begin{aligned}
& T_{3^{n}-1^{n}}=T_{9^{n}-1^{n}}=T_{6^{n}-1^{n}}=[\overline{8}]_{6} \\
& T_{3^{n}-2^{n}}=T_{9^{n}-2^{n}}=T_{6^{n}-2^{n}}=[7,5,1,2,4,8] \\
& T_{3^{n}-4^{n}}=T_{9^{n}-4^{n}}=T_{6^{n}-4^{n}}=[\overline{5,2,8}]_{2} \\
& T_{3^{n}-5^{n}}=T_{9^{n}-5^{n}}=T_{6^{n}-5^{n}}=[4,2,1,5,7,8] \\
& T_{3^{n}-7^{n}}=T_{9^{n}-7^{n}}=T_{6^{n}-7^{n}}=[2,5,8]_{2}
\end{aligned}
$$




$$
\begin{aligned}
& T_{3^{n}-8^{n}}=T_{9^{n}-8^{n}}=T_{6^{n}-8^{n}}=[\overline{1,2}]_{3} \\
& T_{a^{n}-a^{n}}=[\overline{9}]_{9}, T_{2^{n}-1^{n}}=[1,3,7,6,4,9], T_{4^{n}-2^{n}}=[2,3,2,6,2,9] \\
& T_{4^{n}-1^{n}}=[\overline{3,6,9}]_{2}, T_{5^{n}-4^{n}}=[1,9,7,9,4,9], T_{5^{n}-2^{n}}=[3,3,9,6,6,9] \\
& T_{5^{n}-1^{n}}=[4,6,7,3,1,9], T_{7^{n}-5^{n}}=[2,6,2,3,2,9], T_{7^{n}-4^{n}}=[\overline{3,6,9}]_{2} \\
& T_{7^{n}-2^{n}}=[5,9,2,9,8,9], T_{7^{n}-1^{n}}=[\overline{6,3,9}]_{2}, T_{8^{n}-7^{n}}=[1,6,7,3,4,9] \\
& T_{8^{n}-5^{n}}=[3,3,9,6,6,9], T_{8^{n}-4^{n}}=[4,3,7,6,1,9], T_{8^{n}-2^{n}}=[6,6,9,3,3,9] \\
& T_{8^{n}-1^{n}}=[\overline{7,9}]_{3}
\end{aligned}
$$

Table1.5 -The values of $\psi$ of some arithmetic exponential functions

$$
\begin{aligned}
& f(n)=5^{5^{2 n}} \rightarrow \psi_{f(n)}=5, f(n)=5^{5^{2 n+1}} \rightarrow \psi_{f(n)}=2 \\
& f(n)=5^{3^{2 n}} \rightarrow \psi_{f(n)}=5, f(n)=5^{3^{2 n+1}} \rightarrow \psi_{f(n)}=8 \\
& f(n)=7^{3^{n}} \rightarrow \psi_{f(n)}=1, f(n)=2^{2^{2 n+1}} \rightarrow \psi_{f(n)}=4 \\
& f(n)=2^{2^{2 n}} \rightarrow \psi_{f(n)}=7, f(n)=2^{3^{n}} \rightarrow \psi_{f(n)}=8 \\
& f(n)=2^{5^{2 n+1}} \rightarrow \psi_{f(n)}=5, f(n)=2^{5^{2 n}} \rightarrow \psi_{f(n)}=2 \\
& f(n)=5^{2^{2 n}} \rightarrow \psi_{f(n)}=4, f(n)=5^{2^{2 n+1}} \rightarrow \psi_{f(n)}=7 \\
& f(n)=7^{5^{2 n}} \rightarrow \psi_{f(n)}=7, f(n)=7^{5^{2 n+1}} \rightarrow \psi_{f(n)}=4 \\
& f(n)=7^{2^{2 n+1}} \rightarrow \psi_{f(n)}=4, f(n)=7^{2^{2 n}} \rightarrow \psi_{f(n)}=7
\end{aligned}
$$

1.3-Some conclusions that we can reach from the table of periodic matrixes $T_{a^{n} \pm b^{n}}$.

1. The diophantine equation as $z^{n}=x^{n}+y^{n}$ for the some values of $\psi_{z}$ and $\psi_{y}$, on the condition that $x \neq 3 k$, has no solution in the domain of positive integer numbers are as below: $(n \geq 2)$

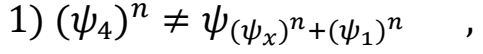
2) $\left(\psi_{1}\right)^{n} \neq \psi_{\left(\psi_{x}\right)^{n}+\left(\psi_{4}\right)^{n}}$
3) $\left(\psi_{5}\right)^{n} \neq \psi_{\left(\psi_{x}\right)^{n}+\left(\psi_{2}\right)^{n}}$,
4) $\left(\psi_{2}\right)^{n} \neq \psi_{\left(\psi_{x}\right)^{n}+\left(\psi_{5}\right)^{n}}$
5) $\left(\psi_{4}\right)^{n} \neq \psi_{\left(\psi_{x}\right)^{n}+\left(\psi_{7}\right)^{n}}$,
6) $\left(\psi_{7}\right)^{n} \neq \psi_{\left(\psi_{x}\right)^{n}+\left(\psi_{4}\right)^{n}}$

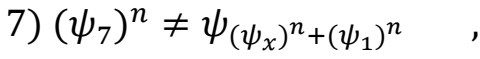
8) $\left(\psi_{1}\right)^{n} \neq \psi_{\left(\psi_{x}\right)^{n}+\left(\psi_{7}\right)^{n}}$
9) $\left(\psi_{8}\right)^{n} \neq \psi_{\left(\psi_{x}\right)^{n}+\left(\psi_{5}\right)^{n}}$,
10) $\left(\psi_{5}\right)^{n} \neq \psi_{\left(\psi_{x}\right)^{n}+\left(\psi_{8}\right)^{n}}$
11) $\left(\psi_{8}\right)^{n} \neq \psi_{\left(\psi_{x}\right)^{n}+\left(\psi_{2}\right)^{n}}$
12) $\left(\psi_{2}\right)^{n} \neq \psi_{\left(\psi_{x}\right)^{n}+\left(\psi_{8}\right)^{n}}$ 


$$
\begin{array}{ll}
\text { 13) }\left(\psi_{8}\right)^{n} \neq \psi_{\left(\psi_{x}\right)^{n}+\left(\psi_{4}\right)^{n}}, \quad \text { 14) }\left(\psi_{8}\right)^{n} \neq \psi_{\left(\psi_{x}\right)^{n}+\left(\psi_{1}\right)^{n}} \\
\text { 15) }\left(\psi_{5}\right)^{n} \neq \psi_{\left(\psi_{x}\right)^{n}+\left(\psi_{1}\right)^{n},} \text { 16) }\left(\psi_{3}\right)^{n} \neq \psi_{\left(\psi_{x}\right)^{n}+\left(\psi_{7}\right)^{n}}
\end{array}
$$

3. When we have $\psi_{a+b} \in\{3,6,9\}$, then all the diophantine equations in the form of $a^{2 n+1}+b^{2 n+1}=(3 c \pm 1)^{2 n+1}$ have no solutions in the domain of positive integers because we can write $\psi_{a^{2 n+1}+b^{2 n+1}} \neq \psi_{(3 c \pm 1)^{2 n-1}}$.

4. When we have $\psi_{a-b} \in\{3,6,9\}$, then $\psi_{a^{n}-b^{n}} \in\{3,6,9\}$ and thus the diophantine equations in the form of $a^{n}=b^{n}+(3 c \pm 1)^{n}$ have no solutions in the domain of positive integer numbers. This is because $\psi_{a^{n}-b^{n}} \neq \psi_{(3 c \pm 1)^{n}}$.

5. $\psi_{a}=2, \psi_{b}=5 \Rightarrow \psi_{a^{2 n-1}+b^{2 n-1}}=7, \psi_{a^{2 n}+b^{2 n}}=2$

6. $\left(\psi_{a}, \psi_{b}\right) \in\{(1,4),(1,7)\} \Rightarrow \psi_{a^{3 n+1}+b^{3 n+1}}=\psi_{a}+\psi_{b}$

7. $\left(\psi_{a}, \psi_{b}\right) \in\{(1,4),(1,7),(4,7)\} \Rightarrow \psi_{a^{3 n}+b^{3 n}}=2$

8. Whenever $a+b=9 k$, then $\psi_{a^{2 n+1}+b^{2 n+1}}=9$. ( $n$ is a natural number)

We just need to use the identity $a^{2 n+1}+b^{2 n+1}=(a+b)\left(a^{2 n}-a^{2 n-1} b^{1}+\cdots+b^{2 n}\right)$ to prove it.

9. The correctness of the congruences below can be easily shown by the properties of the $\psi$ function :( $n_{1}$ and $n_{2}$ are natural numbers)

$$
\begin{aligned}
& \left(9 k_{1}+2\right)^{6 n_{1}-1}+\left(9 k_{2}+2\right)^{6 n_{2}+1} \equiv 7 \bmod 9 \\
& \left(9 k_{1}+5\right)^{6 n_{1}-1}+\left(9 k_{2}+5\right)^{6 n_{2}+1} \equiv 7 \bmod 9 \\
& \left(9 k_{1}+7\right)^{6 n_{1}-1}+\left(9 k_{2}+7\right)^{6 n_{2}+1} \equiv 2 \bmod 9 \\
& \left(9 k_{1}+4\right)^{6 n_{1}-1}+\left(9 k_{2}+4\right)^{6 n_{2}+1} \equiv 2 \bmod 9 \\
& \left(9 k_{1}+8\right)^{6 n_{1}-1}+\left(9 k_{2}+8\right)^{6 n_{2}+1} \equiv 7 \bmod 9 \\
& \left(9 k_{1}+1\right)^{6 n_{1}-1}+\left(9 k_{2}+1\right)^{6 n_{2}+1} \equiv 2 \bmod 9 \\
& \left(6 n_{1}-1\right)^{3}+\left(6 n_{2}+1\right)^{3} \equiv 0 \bmod 9
\end{aligned}
$$

\section{3- The important properties of periodic matrixes of arithmetic functions}

1. If $f(n)$ is an arithmetic polynomial function, its periodic matrix has 9 members and we get:

$$
T_{f(n)}=\left[a_{1}, a_{2}, \ldots, a_{9}\right] \Rightarrow T_{f(n)}=T_{f(n+9 k)}
$$


2. If $f(n)$ is an exponential function or a combination of some arithmetic exponential functions, its periodic matrix has 6 members and we get:

$T_{f(n)}=\left[a_{1}, a_{2}, \ldots, a_{6}\right] \Rightarrow T_{f(n)}=T_{f(n+6 k)}$

3. If $f(n)$ is a combination of some polynomial and exponential functions, then its periodic matrix must have 18 members. (The smallest common multiple of 6 and 9)

$T_{f(n)}=\left[a_{1}, a_{2}, \ldots, a_{18}\right] \Rightarrow T_{f(n)}=T_{f(n+18 k)}$

Here we define two useful sets as $H_{f(n) \oplus g(n)}$ and $H_{f(n) \otimes g(n)}$ for two arbitrary arithmetic functions of $f(n)$ and $g(n)$ as bellow:

$H_{f(n)} \times H_{g(n)}=\left\{\left(\psi_{x}, \psi_{y}\right) \mid \psi_{x} \in H_{f(n)}, \psi_{y} \in H_{g(n)}\right\} \Rightarrow$

$H_{f(n) \oplus g(n)}=\left\{\psi \mid \psi=\psi_{\psi_{x}+\psi_{y}}\left(\psi_{x}, \psi_{y}\right) \in H_{f(n)} \times H_{g(n)}\right\}$

$H_{f(n) \otimes g(n)}=\left\{\psi \mid \psi=\psi_{\psi_{x} \times \psi_{y}}\left(\psi_{x}, \psi_{y}\right) \in H_{f(n)} \times H_{g(n)}\right\}$

As an example, for $H_{g(n)}=\{7,5,8\}$ and $H_{f(n)}=\{2,3\}$ we have:

$H_{f(n)} \times H_{g(n)}=\{(2,7),(2,5),(3,7),(2,8),(3,5),(3,8)\} \Rightarrow$

$H_{f(n) \oplus g(n)}=\left\{\psi_{\psi_{2}+\psi_{7}}, \psi_{\psi_{2}+\psi_{5}}, \psi_{\psi_{2}+\psi_{8}}, \psi_{\psi_{3}+\psi_{7}}, \psi_{\psi_{3}+\psi_{5}}, \psi_{\psi_{3}+\psi_{8}}\right\}$

$=\{9,7,1,1,8,2\}$

Since sequence and repetition of members in a set is meaningless, thus we will get:

$H_{f(n) \oplus g(n)}=\{9,7,1,8,2\}$

As an example of $H_{f(n) \otimes g(n)}$ if we propose $H_{f(n)}=\{3,6\}$ and $H_{g(n)}=\{4,5,7\}$ then we should have:

$H_{f(n)} \times H_{g(n)}=\{(3,4),(3,5),(3,7),(6,4),(6,5),(6,7)\} \Rightarrow$

$H_{f(n) \otimes g(n)}=\left\{\psi_{\psi_{3} \times \psi_{4}}, \psi_{\psi_{3} \times \psi_{5}}, \psi_{\psi_{3} \times \psi_{7}}, \psi_{\psi_{6} \times \psi_{4}}, \psi_{\psi_{6} \times \psi_{5}}, \psi_{\psi_{6}+\psi_{7}}\right\}$

$=\left\{\psi_{12}, \psi_{35}, \psi_{21}, \psi_{24}, \psi_{30}, \psi_{42}\right\}=\{3,8,3,6,3,6\}=\{3,8,6\}$

For both of the arithmetic functions of $f(n)$ and $g(n)$, the periodic matrixes of $T_{f(n)+g(n)}$ and $T_{f(n) \times g(n)}$ will be defined as follows:

$T_{f(n)+g(n)}=\left[\psi_{f(1)+g(1)}, \psi_{f(2)+g(2)}, \ldots, \psi_{f(t)+g(t)}\right]$

$=\left[\psi_{\psi_{f(1)}+\psi_{g(1)}}, \psi_{\psi_{f(2)}+\psi_{g(2)}}, \ldots, \psi_{\psi_{f(t)}+\psi_{g(t)}}\right]$ 


$$
\begin{aligned}
& T_{f(n) \times g(n)}=\left[\psi_{f(1) \times g(1)}, \psi_{f(2) \times g(2)}, \ldots, \psi_{f(t) \times g(t)}\right] \\
& =\left[\psi_{\psi_{f(1)} \times \psi_{g(1)}}, \psi_{\psi_{f(2)} \times \psi_{g(2)}}, \ldots, \psi_{\psi_{f(t)} \times \psi_{g(t)}}\right]
\end{aligned}
$$

\section{The $\psi$ Test}

\section{1- The $\psi$ test and some of its uses}

Since the two sides of algebraic equalities, including equations or identities, have equal value of $\psi$, thus we will define a test that called it the $\psi$ test.

The definition of the $\boldsymbol{\psi}$ test: In every algebraic equality in the form of $P=Q$ in which $P$ and $Q$ are arithmetic functions, the values of $\psi$ of the two arithmetic functions have to be equal. $\left(\psi_{P}=\psi_{Q}\right)$ Therefore, if $H_{P} \cap H_{Q}=\varnothing$ it means the impossibility of that algebraic equality. With the help of $\psi$ test we can prove the incorrectness and impossibility of many algebraic equalities in form of $P=Q$. This test also helps us in proving the unsolvability of many diophantine equations in the domain of integer numbers. The arithmetic function of $\psi$ in algebraic equalities operates similar to relations in physics. In physics, the two sides of a formula or relation have to be dimensionally equal. Similarly, in math and particularly algebra, the value of $\psi$ for two sides of a relation or equation have to be equal.

Theorem 2.1- Whenever gcd $(a, b, c)=1$ and none of the natural numbers of $a, b$, and $c$ are not multiple of 3 , thus the diophantine equation of $a^{n}+b^{n}=c^{n}$ for $n \in\{2,3,4,6\}$ has no solution in the domain of natural numbers.

Proof: Since $a, b$, c are not multiple of 3 , thus we can assume:

$$
a=3 k_{1} \pm 1 \quad, \quad b=3 k_{2} \pm 1 \quad, \quad c=3 k_{3} \pm 1
$$

Therefore, according to the table of $\psi$ values for arithmetic functions of $f(n)=$ $(3 k \pm 1)^{n}$ and $f(n)=(3 k)^{n}$, we have:

$$
\begin{aligned}
& n=2 \Rightarrow\left\{\begin{array}{l}
\psi_{a^{2}+b^{2}} \in\{2,5,8\} \\
\psi_{c^{2}} \in\{1,4,7\}
\end{array} \Rightarrow \psi_{a^{2}+b^{2}} \neq \psi_{c^{2}}\right. \\
& n=3 \Rightarrow\left\{\begin{array}{l}
\psi_{a^{3}+b^{3}} \in\{2,7,9\} \\
\psi_{c^{3}} \in\{1,8\}
\end{array} \Rightarrow \psi_{a^{3}+b^{3}} \neq \psi_{c^{3}}\right. \\
& n=4 \Rightarrow\left\{\begin{array}{l}
\psi_{a^{4}+b^{4}} \in\{2,5,8\} \\
\psi_{c^{4}} \in\{1,4,7\}
\end{array} \Rightarrow \psi_{a^{4}+b^{4}} \neq \psi_{c^{4}}\right. \\
& n=6 \Rightarrow\left\{\begin{array}{l}
\psi_{a^{6}+b^{6}} \in\{2\} \\
\psi_{c^{6}} \in\{1\}
\end{array} \Rightarrow \psi_{a^{6}+b^{6}} \neq \psi_{c^{6}}\right.
\end{aligned}
$$

As a result, the proof is complete. 
Thus, according to the theorem (8)and propose $n_{1}, n_{2}, n_{3}$ are non-negative integer numbers,

in the general case, we get:

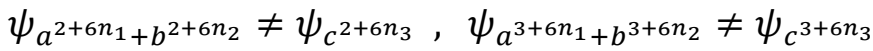

$\psi_{a^{4+6 n_{1}}+b^{4+6 n_{2}}} \neq \psi_{c^{4+6 n_{3}}}, \psi_{a^{6 n_{1}}+b^{6 n_{2}}} \neq \psi_{c^{6 n_{3}}}$

Theorem 2.2- Whenever we assume gcd $(a, b, c)=1$, then the below diophantine equations have no solutions in the domain of natural numbers. $\left(n_{1}, n_{2}, n_{3}\right.$ are nonnegative integer numbers.)

1) $a^{2+6 n_{1}}+b^{2+6 n_{2}}=(3 c)^{2+6 n_{3}}$, 2) $a^{4+6 n_{1}}+b^{4+6 n_{2}}=(3 c)^{4+6 n_{3}}$

3) $a^{6 n_{1}}+b^{6 n_{2}}=(3 c)^{6 n_{3}}$

Proof: According to the table of values of $\psi$ for the arithmetic functions of $f(n)=$ $(3 k \pm 1)^{n}$ and $f(n)=(3 k)^{n}$ and the theorem (1.8), we have:

$\psi_{a^{2}+b^{2}} \in\{2,5,8\}, \psi_{(3 c)^{2}} \in\{9\} \Rightarrow \psi_{a^{2}+b^{2}} \neq \psi_{(3 c)^{2}} \Rightarrow \psi_{a^{2+6 n_{1}+b^{2+6 n_{2}}}} \neq \psi_{(3 c)^{2+6 n_{3}}}$

$\psi_{a^{4}+b^{4}} \in\{2,5,8\}, \psi_{(3 c)^{4}} \in\{9\} \Rightarrow \psi_{a^{4}+b^{4}} \neq \psi_{(3 c)^{4}} \Rightarrow \psi_{a^{4+6 n_{1}}+b^{4+6 n_{2}}} \neq \psi_{(3 c)^{4+6 n_{3}}}$

$\psi_{a^{6}+b^{6}} \in\{2\}, \psi_{(3 c)^{6}} \in\{9\} \Rightarrow \psi_{a^{6}+b^{6}} \neq \psi_{(3 c)^{6}} \Rightarrow \psi_{a^{6 n_{1}+b^{6 n_{2}}}} \neq \psi_{(3 c)^{6 n_{3}}}$

Example 2.1- Show that the diophantine equation of $x^{2}-9 y^{2}=5$ has no solution in the domain of natural numbers.

Answer: $x^{2}-9 y^{2}=5 \Rightarrow x^{2}=9 y^{2}+5$

$\psi_{x^{2}} \in\{1,4,7,9\}=H_{x^{2}}=H_{y^{2}} \quad, \quad \psi_{9 y^{2}} \in\{9\} \Rightarrow H_{5 \oplus 9 y^{2}}=\{5\}$

As a result, we have:

$H_{x^{2}} \neq H_{5 \oplus 9 y^{2}} \Rightarrow \psi_{9 y^{2}+5} \neq \psi_{x^{2}}$

The diophantine equation of $x^{2}-9 y^{2}=8$, similar to the previous example, has no solution in the domain of natural numbers.

Theorem 2.3- Show that the diophantine equation of $x^{2}+y^{2}=z^{2}$ in the domain of natural numbers has solution if $x$ or $y$ is a multiple of 3 .

Proof: State 1- If $x, y, z$ are not multiple of 3.

$H_{x^{2}}=H_{y^{2}}=H_{z^{2}}=\{1,4,7,9\} \Rightarrow H_{x^{2} \oplus y^{2}}=\{2,5,8\} \Rightarrow H_{x^{2} \oplus y^{2}} \cap H_{z^{2}}=\phi$

State 2-If only $z$ is a multiple of 3. 
$z=3 k \Rightarrow H_{z^{2}}=\{9\} \Rightarrow H_{x^{2} \oplus y^{2}} \cap H_{z^{2}}=\phi$

State 3-If $x$ or $y$ are multiples of 3. Then in this case we get:

$H_{y^{2}}=\{9\} \Rightarrow H_{x^{2} \oplus y^{2}} \cap H_{z^{2}}=\{1,4,7,9\} \neq \phi$

Example 2.2 -Show that the diophantine equation of $2 x^{3}+5 y^{3}=431$ in the domain of natural numbers can not have a solution.

Answer:

$H_{x^{3}}=H_{y^{3}}=\{1,8,9\} \Rightarrow\left\{\begin{array}{l}H_{2 x^{3}}=\{2,7,9\} \\ H_{5 y^{3}}=\{5,4,9\}\end{array} \Rightarrow H_{2 x^{3} \oplus 5 y^{3}}=\{2,3,4,5,6,7,9\}\right.$

Since $\psi_{431}=8$, thus $\psi_{2 x^{3}+5 y^{3}} \neq \psi_{431}$. This means that the above equation in the domain of natural numbers can not have a solution.

Example 2.3- Show that the diophantine equation of $8^{x}+7^{y}=19^{Z}$ in the domain of natural numbers has no solution.

Answer: $H_{8^{x}}=\{1,8\}, H_{7^{y}}=\{1,4,7\} \Rightarrow H_{8^{x} \oplus 7^{y}}=\{2,5,8,9,3,6\}$

$H_{19^{z}}=\{1\} \Rightarrow H_{8^{x} \oplus 7^{y}} \cap H_{19^{z}}=\varnothing$

Theorem 2.4- The diophantine equation of $x^{6}=y^{4}+z^{4}$ in the domain of positive integer numbers has no solution when we assume $\operatorname{gcd}(x, y, z)=1$.

Proof: State 1-If $x, y, z$ are not multiples of 3. In other words, if we have:

$x=3 k_{1} \pm 1, y=3 k_{2} \pm 1, z=3 k_{3} \pm 1$

$\Rightarrow H_{z^{4}}=H_{y^{4}}=\{1,4,7\} \Rightarrow H_{y^{4} \oplus z^{4}}=\{2,5,8\}$.

Since we have $H_{x^{6}}=\{1\}$ therefore:

$H_{x^{6}} \cap H_{y^{4} \oplus z^{4}}=\emptyset \Rightarrow \psi_{x^{6}} \neq \psi_{y^{4}+z^{4}}$

State 2-If we assume $z=3 k_{3}$ then we can write:

$x^{6}=y^{4}+z^{4} \Rightarrow\left(x^{3}\right)^{2}=\left(y^{2}\right)^{2}+\left(z^{4}\right)$

From pythagorean numbers we have:

$$
\begin{aligned}
& \mid \begin{array}{l}
x^{2}=m^{2}+n^{2} \\
y^{2}=m^{2}-n^{2} \\
z^{2}=2 m n
\end{array} \\
& z=3 k_{3} \Rightarrow z^{2}=9 k^{2}=\left.2 m n \Rightarrow\right|_{n=9 r} ^{m=9} \pm 1
\end{aligned}
$$


